\title{
The selective neurotoxin DSP-4 impairs the noradrenergic projections from the locus coeruleus to the inferior colliculus in rats
}

\author{
Sebastián Hormigo ${ }^{1,2}$, José de Anchieta de Castro e Horta Júnior ${ }^{3}$, Ricardo Gómez-Nieto ${ }^{1,2,4}$ and \\ Dolores E. López ${ }^{1,2,4}$ *
}

1 Institute for Neuroscience of Castilla y Leon, University of Salamanca, Salamanca, Spain

2 Institute of Biomedical Research of Salamanca, University of Salamanca, Salamanca, Spain

${ }^{3}$ Biosciences Institute - Campus of Botucatu, São Paulo State University - UNESP-, São Paulo, Brazil

${ }^{4}$ Department of Cellular Biology and Pathology, University of Salamanca, Salamanca, Spain

Edited by:

Eric D. Young, Johns Hopkins

University, USA

\section{Reviewed by:}

Douglas L. Oliver, University of

Connecticut Health Center, USA

Laura M. Hurley, Indiana University,

USA

\section{${ }^{*}$ Correspondence:}

Dolores E. López, Institute for

Neuroscience of Castilla y Leon (INCYL), University of Salamanca, C/ Pintor Fernando Gallego, 1, 37007 Salamanca, Spain. e-mail:lopezde@usal.es
The inferior colliculus (IC) and the locus coeruleus (LC) are two midbrain nuclei that integrate multimodal information and play a major role in novelty detection to elicit an orienting response. Despite the reciprocal connections between these two structures, the projection pattern and target areas of the $\mathrm{LC}$ within the subdivisions of the rat $\mathrm{IC}$ are still unknown. Here, we used tract-tracing approaches combined with immunohistochemistry, densitometry, and confocal microscopy (CM) analysis to describe a projection from the LC to the IC. Biotinylated dextran amine (BDA) injections into the LC showed that the LC-IC projection is mainly ipsilateral $(90 \%)$ and reaches, to a major extent, the dorsal and lateral part of the IC and the intercollicular commissure. Additionally, some LC fibers extend into the central nucleus of the IC. The neurochemical nature of this projection is noradrenergic, given that tyrosine hydroxylase (TH) and dopamine beta hydroxylase (DBH) colocalize with the BDA-labeled fibers from the LC. To determine the total field of the LC innervations in the IC, we destroyed the LC neurons and fibers using a highly selective neurotoxin, DSP-4, and then studied the distribution and density of $\mathrm{TH}$ - and DBH-immunolabeled axons in the IC. In the DSP-4 treated animals, the number of axonal fibers immunolabeled for $\mathrm{TH}$ and $\mathrm{DBH}$ were deeply decreased throughout the entire rostrocaudal extent of the IC and its subdivisions compared to controls. Our densitometry results showed that the IC receives up to $97 \%$ of its noradrenergic innervations from the LC neurons and only $3 \%$ from non-coeruleus neurons. Our results also indicate that TH immunoreactivity in the IC was less impaired than the immunoreactivity for DBH after DSP-4 administration. This is consistent with the existence of an important dopaminergic projection from the substantia nigra to the IC. In conclusion, our study demonstrates and quantifies the noradrenergic projection from the $\mathrm{LC}$ to the $\mathrm{IC}$ and its subdivisions. The re-examination of the $\mathrm{TH}$ and DBH immunoreactivity after DSP-4 treatment provides insights into the source, extent, and topographic distribution of the LC efferent network in the IC, and hence, contributes to our understanding of the role of the noradrenaline (NA) system in auditory processing.

Keywords: biotinylated dextran amine, dopamine beta hydroxylase deficiency, immunohistochemistry, densitometry, neurotoxicity, noradrenergic efferents, noradrenergic modulation, tyrosine hydroxylase

\begin{abstract}
Abbreviations: $4 \mathrm{~V}$, forth ventricle; $5 \mathrm{n}$, trigeminal nerve; $\mathrm{ABC}$, avidin biotinperoxidase complex; Aq, Aqueduct (Sylvius); BDA, biotinylated dextran amine; $\mathrm{CM}$, confocal microscopy; $\mathrm{CN}$, cochlear nucleus; CNIC, central nucleus of the inferior colliculus; DAB, 3,3' diaminobenzidine; DBH, dopamine beta hydroxylase; DCIC, dorsal cortex of the inferior colliculus; DNLL, dorsal nucleus of the lateral lemniscus; DSP-4, N-(2-chloroethyl)-N-ethyl-2-bromobenzylamine; LCIC, lateral cortex of the inferior colliculus; IA, Interaural level; IC, inferior colliculus; IHC, immunohistochemistry; ir, immunoreactivity; IS, injection site; LC, locus coeruleus; LM, light microscopy; ml, medial lemniscus; MNTB, medial nucleus of the trapezoid body; PB, phosphate buffer $0.1 \mathrm{M}, \mathrm{pH}$ 7.4; PBS, phosphate buffer saline; PnO, pontine reticular nucleus, oral part; NA, noradrenaline; TH, tyrosine hydroxylase; Tx, Triton X100; tz, trapezoid body; VNLL, ventral nucleus of the lateral lemniscus.
\end{abstract}

\section{INTRODUCTION}

The locus coeruleus (LC) is a structure located in the caudal central gray pons under the floor of the fourth ventricle. It was first described in 1809 by the German neuroanatomist Johann C. Reil, but it was not until 1812 that the Wenzel brothers coined the name (Toshihiro, 2000). The LC is involved in many of the sympathetic effects during stress due to an increased production of noradrenaline (NA). Electrophysiological studies support the hypothesis that the LC is activated by various stressful stimuli (Valentino et al., 1983; Page et al., 1992; Curtis et al., 1993; del C. Gonzalez et al., 1995; Singewald et al., 1995; Conti and 
Foote, 1996; Dawe et al., 2001; Valentino and Van Bockstaele, 2008), noxious/nociceptive stimuli (Singewald et al., 1995; Couto et al., 2006; Viisanen and Pertovaara, 2007; Liu et al., 2008; Imbe et al., 2009; Tsuruoka et al., 2011), and physiological stimuli, such as hypotension, hypoxia, and visceral stimulation, which increase the firing of the neurons in this structure (Miao-Kun, 1995; Singewald et al., 1995; Lechner et al., 1997; Valentino et al., 2005; Ma et al., 2008).

The LC is the main source of NA for both the brainstem and the forebrain (Aston-Jones, 2004). Previous investigations have described the catecholamine biosynthetic pathways and their regulation in the nervous system. The metabolic pathways can be finely regulated when there is an increase in the demand of synthesis for NA, for instance in a stressful situation or in the detection of novelty stimuli (Potter et al., 1962; Snyder et al., 1965; Axelrod et al., 1969; Ciaranello et al., 1976).

Studies have shown that the neurotoxin N-(2-chloroethyl)-Nethyl-2-bromobenzylamine (DSP-4) can be used for the temporary selective degradation of the central and peripheral noradrenergic neurons, mainly those from the LC (Fritschy and Grzanna, 1989; Grzanna et al., 1989; Fritschy et al., 1991). Based on these experiments, the authors described the existence of two separate subsystems of noradrenergic fibers, the LC and non-coerulean noradrenergic fibers, which differ not only in their projections but also with respect to the pharmacological properties of their axon terminals. The neurotoxin DSP-4 exclusively affects the noradrenergic fibers from the LC (Fritschy and Grzanna, 1989; Grzanna et al., 1989). It has been suggested that this selectivity of DSP-4 toward LC neurons may be related to the significant difference found in the affinity of DSP-4 for the NA uptake carrier in different brain structures (Zaczek et al., 1990). In rodents, a systemic injection of DSP-4 causes a depletion in the levels of NA, in the release capacity and in the activity of dopamine beta hydroxylase (DBH) (Ross et al., 1973; Ross, 1985). The effects of DSP-4 administration on the behavior of rats (open-field tests) can include neophobia (distorted reactions to new things or experiences), increased emotionality (more grooming activities and number of stools), defensive or submissive behavior, an altered resident/intruder paradigm, increased aggressiveness and a lack of fear to environmental factors (Spyraki et al., 1982; Delini-Stula et al., 1984; Cornwell-Jones et al., 1992; Harro et al., 1995; van den Buuse et al., 2001).

With regard to the auditory pathway, the LC provides noradrenergic inputs to the cochlear nuclei (Klepper and Herbert, 1991; Ebert, 1996; Thompson, 2003), the superior olivary complex (Mulders and Robertson, 2001), the cochlear root nucleus (Gómez-Nieto et al., 2008b) and the inferior colliculus (IC) (Klepper and Herbert, 1991). The IC is an important auditory nucleus in the brainstem with significant physiological implications, not only for hearing processing but also for adaptive mechanisms, such as the acoustic startle reflex (Jordan and Leaton, 1982; Leitner and Cohen, 1985; Li et al., 1998; Li and Yeomans, 2000; Li and Yue, 2002; Heldt and Falls, 2003; Nobre et al., 2003; Silva et al., 2005; Reimer et al., 2008; Satake et al., 2012). The LC and the IC are reciprocally connected (Klepper and Herbert, 1991; Freitas et al., 2006). Nevertheless, little is known about the projection pattern and target areas of the LC within the IC. The aim of this study was to investigate the noradrenergic innervation of the LC throughout the rostrocaudal extent of the IC and its subdivisions. We attempt to reveal the extensive LC noradrenergic efferent network within the IC using anterograde tract-tracing techniques combined with immunohistochemistry in addition to densitometry and colocalization studies of the catecholaminergic enzymes in control and DSP- 4 treated rats. Based on the hypothesis that there are two separate subsystems of noradrenergic fibers and that the neurotoxin DSP-4 exclusively affects the noradrenergic fibers from the LC (Fritschy and Grzanna, 1989; Grzanna et al., 1989), we used DSP-4 as a highly selective neurotoxin to eliminate the projections from the LC. Using this approach, we studied the changes in DBH immunohistochemistry in the IC before and after DSP- 4 treatment to evaluate the loss of the LC noradrenergic projections to the IC and quantified the difference.

\section{MATERIALS AND METHODS ANIMALS AND EXPERIMENTAL DESIGN}

Seventeen adult albino rats (Wistar strain) weighting 250-300 g were used (Charles River, Barcelona, Spain). The experiments were conducted according to the guidelines for the use and care of laboratory animals of the European Communities Council Directive (DOCE L222; 24-08-1999) and with those established by the Spanish Government (RD 1201/2005). Animals were housed in groups of two or three prior to surgery and individually afterwards. All efforts were made to minimize the number of animals that were used and their suffering.

We designed a prospective study. In the first step, we studied the projections from the LC to the IC. Then, we demonstrated the noradrenergic nature of these projections in the IC through spatial overlapping techniques, and we described the noradrenergic immunoreactivity in the IC. Afterwards, we investigated the morphological modifications in animals treated with the neurotoxin DSP-4.

\section{INJECTION OF NEURONAL TRACERS}

To study the neuronal projections from the LC to the IC, we injected nine animals with biotinylated dextran amine (BDA, 10,000 MW, Molecular Probes, Eugene, OR, USA; 10\% in distilled water) in the left LC. The neuronal tracer BDA was injected after deep anesthetization of the animal through an intramuscular administration of a mixture of xylazine $(7 \mathrm{mg} / \mathrm{kg})$ and ketamine (40 mg/kg). When necessary, additional anesthesia (one-fifth of the initial dose) was given during surgery. Each animal was placed in a stereotaxic frame (Kopf, Tujunga, CA, USA) using the headpositioning procedure described by Paxinos and Watson (2005). The skull was exposed by a midline scalp incision, and a craniotomy was performed over the injection coordinates (Fritschy and Grzanna, 1990). Because the LC is topographically organized, the injection sites were preferentially located in and around the dorsal half of the nucleus, where the ascending projections originate (Guyenet, 1980; Fritschy and Grzanna, 1990). BDA was injected iontophoretically via a glass micropipette $(25 \mu \mathrm{m}$ tip diameter) with $3 \mu \mathrm{A}$ positive current pulses ( $7 \mathrm{~s}$ on/7s off) for $15 \mathrm{~min}$. Following the tracer injection, the pipette was left in place for $15 \mathrm{~min}$ without current passing to prevent the backflow, then 
the scalp was sutured and the animal was allowed to recover for a minimum of 7 d (Gómez-Nieto et al., 2008a; Horta-Júnior et al., 2008).

\section{VISUALIZATION OF NEUROTRACERS}

At the end of the survival period, the animals were euthanized with a sodium pentobarbital overdose and then perfused transcardially with $100 \mathrm{ml}$ of fresh Ringer calcium-free buffer $(\mathrm{NaCl}$, $145.45 \mathrm{mM}$; KCl, $3.35 \mathrm{mM}$; $\mathrm{NaHCO}_{3}, 2.38 \mathrm{mM}$ ), pH 6.9, at $37^{\circ} \mathrm{C}$, followed by $1000 \mathrm{ml}$ of fresh depolymerized $4 \%$ paraformaldehyde in $0.1 \mathrm{M}$ phosphate buffer, $\mathrm{pH} 7.4(\mathrm{~PB})$, at room temperature. For either light microscopy (LM) or confocal microscopy (CM), the brains were removed and cryoprotected for $48 \mathrm{~h}$ at $4^{\circ} \mathrm{C}$ in $30 \%$ sucrose in PB. Serial coronal sections ( $40 \mu \mathrm{m}$ in thickness) were cut on a freezing stage sliding microtome (HM430; Microm, Heidelberg, Germany). The serial sections were collected in PB and divided into a series of 10 bins.

For LM, the tracers were visualized using a peroxide-based protocol with Tris-buffered saline containing $0.2 \%$ Triton X-100 (TBS-TX, \#T9284; Sigma, St. Louis, MO, USA) in washing steps. To reveal the BDA tracer, free-floating sections were incubated with an avidin biotin-peroxidase complex (ABC, Standard-kit \#PK 4000; Vector Laboratories, Burlingame, CA, USA) for $2 \mathrm{~h}$ and visualized with a 3,3' diaminobenzidine tetrahydrochloride (DAB) and nickel ammonium sulfate solution (Hancock, 1982; Hsu and Soban, 1982).

\section{TH AND DBH IMMUNOHISTOCHEMISTRY FOR LM}

To study the pattern of $\mathrm{TH}$ and $\mathrm{DBH}$ immunoreactivity, we used eight animals. After the perfusion and serial section protocol, standard immunostaining procedures were followed for the respective sections, which entailed incubating the sections for $72 \mathrm{~h}$ at $4^{\circ} \mathrm{C}$ in a primary antibody solution diluted in TBS-Tx. The tissue was then washed and incubated in its corresponding biotinylated secondary antibody for $2 \mathrm{~h}$ at room temperature. The antibodies used and their dilution are shown in Table $\mathbf{1}$.

Following the removal of the secondary antibodies, the sections were reacted with $\mathrm{ABC}$ as described above, using $\mathrm{DAB}$ as the chromogen. All sections were mounted on slides, dehydrated and coverslipped with Entellan ${ }^{\circledR}$ Neu (Merck, Darmstadt, Germany).
In addition, several series were counterstained with cresyl violet $0.1 \%$ (\#5235, Merck) for cytoarchitectonic reference, chiefly to identify the subdivisions of the IC.

\section{COLOCALIZATION EXPERIMENTS}

To demonstrate the noradrenergic nature of the BDA-labeled fibers from the LC, we have performed experiments to highlight the colocalization between, tyrosine hydroxylaseimmunoreactivity (TH-ir) and dopamine beta hydroxylaseimmunoreactivity (DBH-ir) in the IC using CM. Free-floating sections were pretreated with phosphate buffer saline (PBS) $0.3 \%$ Triton X-100 and blocked for $1 \mathrm{~h}$ at room temperature with 6\% normal goat serum (\#S-1000, Vector Laboratories) in PBS. After overnight incubation at $4^{\circ} \mathrm{C}$ with primary antibodies, the sections were washed in PBS and incubated for $1 \mathrm{~h}$ at room temperature with the secondary antibody (Table 1). The BDA was revealed by incubation in Cy2-conjugated streptavidin (1/650; \#016-220-084; Jackson ImmunoResearch). Later on, the sections were rinsed, mounted on slides and coverslipped with the ProLong ${ }^{\circledR}$ Antifade kit (\#P7481; Molecular Probes).

In all immunohistochemistry experiments, the omission of the primary antibody resulted in no staining (see Results for more details).

\section{NEUROTOXIC LESION WITH DSP-4}

To study the TH-ir and DBH-ir fibers located in the IC following the administration of DSP-4 (Sigma-Aldrich Co, St. Louis, MO, USA), we performed immunohistochemistry for $\mathrm{TH}$ and $\mathrm{BDH}$ in five animals treated with DSP-4 and five control animals. We administrated a single intraperitoneal dose of $50 \mathrm{mg} / \mathrm{kg}$ of DSP-4 in five rats, as previously described (Ross, 1985; Grzanna et al., 1989; Fritschy et al., 1991). After 15 d, we sacrificed the rats.

\section{IMAGE ANALYSIS}

The sections processed for LM were studied using a microscope (\#BX5; Olympus, Center Valley, PA, USA) equipped with a digital camera (Spot $\mathrm{Rt}^{\circledR}$; Diagnostic Instruments, Sterling Heights, MI, USA). Low-magnification images were taken with a $5 \times$ objective lens, and high-magnification images were taken with a $20 \times$ objective lens.

Table 1 | List of antibodies and dilutions used for the immunohistochemical approaches.

\begin{tabular}{|c|c|c|c|c|c|c|c|}
\hline Antigen & Primary antibody & Reference & Dilution & Secondary antibody & Reference & Dilution & Method \\
\hline & & & & Goat Cy5 anti-mouse IgG & \#115-175-003-JI & $1 / 250$ & $\mathrm{CM}$ \\
\hline \multirow[t]{2}{*}{$\mathrm{DBH}$} & Rabbit anti-DBH & \#DZ1020-Affiniti & $1 / 500$ & Biotinylated goat anti-rabbit & \#BA-1000-Vector & $1 / 200$ & LM \\
\hline & & & & Goat Cy5 anti-rabbit lgG & \#111-175-003-Jl & $1 / 250$ & $\mathrm{CM}$ \\
\hline
\end{tabular}

Affinity Research Products, Nottingham, UK; Diasorin, Stillwater, MN, USA; JI: Jackson Immunoresearch, West Grove, PA, USA; Vector Laboratories, Burlingame, CA, USA. The specificity of the anti-TH antibody: the antibody recognizes an epitope present in the N-terminal region (between a 40-152) of both rodent and human tyrosine hydroxylase. It detects both the intact subunits and the 59/57 kDa doublet and an array of TH forms with decreasing molecular weights resulting from severe proteolysis. The specificity of the anti-DBH antibody: the dopamine $\beta$-hydroxylase precursor recombinant protein epitope signature tag (PrEST).

Immunogen sequence: VQRTPEGLTLLFKRPFGTCDPKDYLIEDGTVHLVYGILEEPFRSLEAINGSGLOMGLQRVQLLKPNIPEPELPSDACTMEVQAPNIQIPSQETTYWCYIKELPKGFSRHHIIKYEPIV TKGN. 
The sections processed for CM were first examined using a microscope (\#BX5; Olympus) equipped with epifluorescence and a digital camera (Spot $\mathrm{Rt}^{\circledR}$ ). Then, the sections were processed and analyzed using a Leica TCS SP2 confocal laser scanning microscope (Leica Microsystems, Mannheim, Germany) coupled to a Leica DM IRE2 inverted microscope and equipped with argon and helium neon lasers with excitation wavelengths of 458, 476, $488,543,568$, and $633 \mathrm{~nm}$. The fluorochromes Cy2 and Cy5 were detected sequentially, stack-by-stack, with the acousto-optical tunable filter system and triple dichroic mirror TD488/543/633 using laser lines 488-495, 546, and $633 \mathrm{~nm}$. The background was controlled, and the photomultiplier voltage $(800 \mathrm{~V})$ was selected for maximum sensitivity in the linear range. The objectives used were Planapochromat $20 \times$, oil immersion $40 \times$, and $63 \times /$ numerical aperture 1.30 , giving a resolution of $\sim 150 \mathrm{~nm}$ in the xy-plane and $\sim 300 \mathrm{~nm}$ along the $\mathrm{z}$-axis (pinhole 1 Airy unit), as well as several electronic zoom factors up to $1.58 \times$.

All photomicrographs shown in the figures were processed with minor modifications in brightness, contrast and to remove the tissue free background using Adobe Photoshop ${ }^{\circledR}$ (version 9.0; Adobe Systems Incorporated, San Jose, CA, USA). The IC fiber drawing was made using Adobe Photoshop ${ }^{\circledR}$ to mount digitalized slices from high-magnification images acquired with $20 \times$ objective lens and Canvas ${ }^{\circledR}$ (version X Build 885, ACD Systems Inc, USA) to draw the labeled fibers as well as the assemblage of all figures.

The densitometry of TH-ir and DBH-ir was made using the Canvas fiber drawings. The immunoreactive fibers in the IC were drawn with $0.176 \mathrm{~mm}$ thick black solid lines on a white background. The quantification of the mean gray level for the subdivisions of the IC in different rostrocaudal levels was performed on traces of stained sections using ImageJ software (version 1.43, Rasband, W.S., ImageJ, US National Institutes of Health, Bethesda, Maryland, USA, http://imagej.nih.gov/ij/, 1997-2011).

Different experimental conditions were compared using the unpaired Student's $t$-test. Data are reported as the means \pm SD. The statistical package we employed was PASW Statistics 18.0.0 (SPSS Inc., Chicago, IL, USA). Statistical significance was accepted at $p<0.05$.

\section{RESULTS}

\section{LC SENDS PROJECTIONS TO THE IC}

The tracer injection experiments resulted in a single BDA injection in the left LC (Figure 1A). The injection sites were elliptical with a typical size of $\sim 600 \times 600 \mu \mathrm{m}$ in the rostrocaudal and mediolateral axis, respectively. We only used those injections that did not spread to adjacent nuclei, e.g., to the laterodorsal tegmental nucleus (LDTg). The injections were restricted to the nuclear core of the LC. We also detected anterograde labeling in known LC targets, including the dorsal raphe (Kim et al., 2004), and brain structures of the somatosensory pathway, such as the somatosensory cortex, the ventrobasal nucleus of the thalamus, and the principal nucleus of the trigeminal nerve (Simpson et al., 1997). In all cases, we observed thick $(1.04 \pm 0.78 \mu \mathrm{m})$, straight BDAlabeled fibers with varicosities en passage (beaded fibers), and thin $(0.8 \pm 0.15 \mu \mathrm{m})$, branching BDA-labeled fibers with buttonlike varicosities (terminal plexus) in both the ipsilateral and contralateral IC (Figures 1B,C,D, and E). These terminal fibers were located predominantly in the lateral and dorsal cortices and in the intercollicular commissure.

The LC-IC projection was mainly ipsilateral ( $90 \%)$, although each LC directs some fibers to the contralateral IC $(\sim 10 \%)$ (Figure 1F). We found a few labeled fibers from the LC in the central nucleus of the IC.

\section{NEUROCHEMICAL IDENTITY OF LC TERMINAL FIBERS IN THE IC}

Fibers that were immunoreactive for $\mathrm{TH}$ and $\mathrm{DBH}$ in the IC were similar in distribution and size to those described for the BDA-labeled fibers from the LC. We observed TH-ir and BDH-ir beaded fibers that branched into thinner $\mathrm{TH}$-ir and $\mathrm{BDH}$-ir fibers with button-like varicosities (Figures 2 B and 3B).

To show that the BDA-labeled endings from the LC contained catecholaminergic enzymes ( $\mathrm{TH}$ and $\mathrm{DBH}$ ), we combined a BDA injection in the LC with immunohistochemistry for TH or $\mathrm{DBH}$. Our results show that BDA-labeled fibers from the LC colocalized with TH-positive fibers and terminals, indicating the catecholaminergic nature of the LC-IC projection (Figure 2). Furthermore, some labeled fibers in the IC from the LC were identified as noradrenergic because we observed colocalization between the BDA-labeled fibers and the DBH-ir fibers (Figure 3).

Despite the high degree of colocalization in the IC between TH-ir and DBH-ir fibers (Figure 4), not every catecholaminergic fiber was noradrenergic in nature. The immunoreactivity for $\mathrm{TH}$ had incomplete spatial overlapping with DBH-ir (Figure 4). This suggests that others structures in the brainstem and in other brain regions are sending dopaminergic projections to the IC.

\section{DSP-4 ADMINISTRATION IMPAIRED PROJECTIONS FROM THE LC TO THE IC}

On average, the weight of the treated rats was approximately $12 \%$ lower than that of control rats at one week post-treatment and approximately 9\% lower than controls at two weeks after the DSP4 administration. The administration of DSP-4 in rats also causes changes in urination (a loss of rhythm patterns of diurnal urination), a large increase in void volume and increased water intake, all of which are due to changes in TH (Ranson et al., 2003). We also observed disturbed sleep in which the sleep-wake cycle was altered during the first five post-treatment but then returned to control values.

To determine the total field of the LC projection to the entire rostrocaudal extent of the IC and its subdivisions, we used $\mathrm{TH}$ and $\mathrm{DBH}$ immunohistochemistry and re-examined the LC-IC projection after DSP-4 administration. In non-treated animals, we found intense immunoreactivity for both $\mathrm{TH}$ and DBH in the LC (Figures 5A and B). Within the LC, we found dark-stained fibers and cell bodies immunolabeled for both enzymes. As described elsewhere (Klepper and Herbert, 1991), we observed this immunolabeled pattern of fibers and cell bodies throughout the rostral-caudal extent of the LC. At 15 days postadministration in DSP-4 rats, the immunoreactivity for those enzymes decidedly decreased. Thus, large projection neurons and the fibers of the LC could no longer be visualized with $\mathrm{TH}$ and DBH immunohistochemistry after DSP-4 treatment (Figures 5C and $\mathbf{D})$. This indicates that the efferents from the LC are affected 


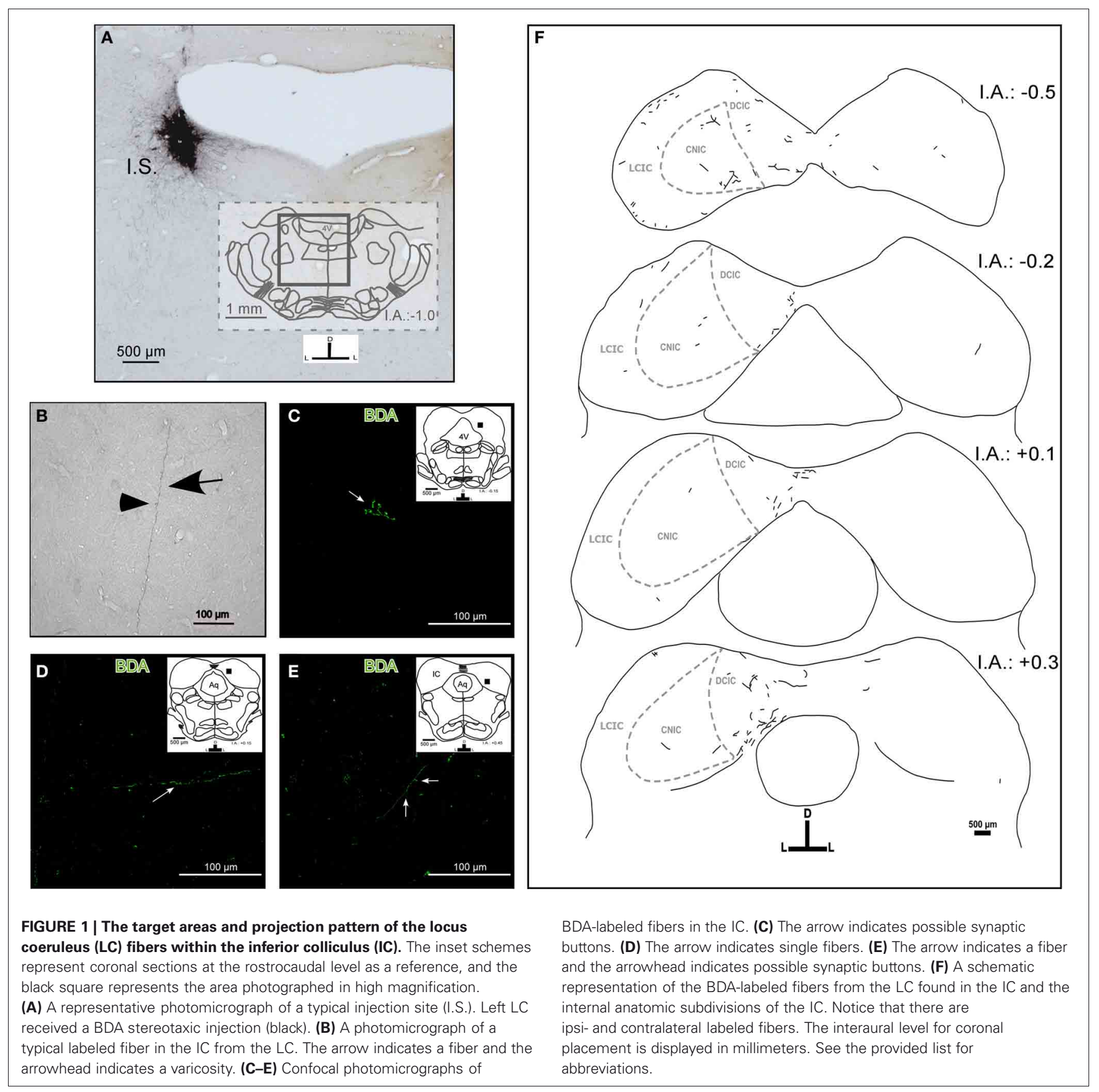

by the action of the drug, including the noradrenergic fibers in the IC, as presented in the following results. The immunohistochemical negative controls (no primary antibody added) showed no immunoreactivity for TH or DBH (Figures 5E,F,G, and $\mathbf{H}$ ).

For a better description of the results, we divided the rostrocaudal extension of the IC into three different parts: caudal, middle, and rostral. We found a dense $\mathrm{TH}$-ir throughout the entire IC in the control animals, with the highest mean gray values at the caudal level, mainly in the DCIC and the CNIC (Figure 6A). The fibers spread homogeneously in the IC, and there was no significant difference in the distribution along the subdivisions $(p>0.05)$. There was a great reduction in TH fibers in all subdivisions of the IC in the animals treated with DSP4 (Figure 6B). The most important diminutions were found in the caudal $(72.9 \%, p<0.01)$ and the middle $(68.8 \%, p<0.01)$ portions of the CNIC and in the whole length of the LCIC (caudal 73\%, middle $72.3 \%$, and rostral $50.9 \%$, for all cases $p<0.01$ ) (Figures 6C,D, and E), whereas the rostral part of the DCIC and the intercollicular commissure remained almost unaltered. The observed reduction was approximately 10\% $(p<0.05)$ (Figure 6E), suggesting that approximately $90 \%$ of the labeled fibers in this region belong to the dopaminergic system. 

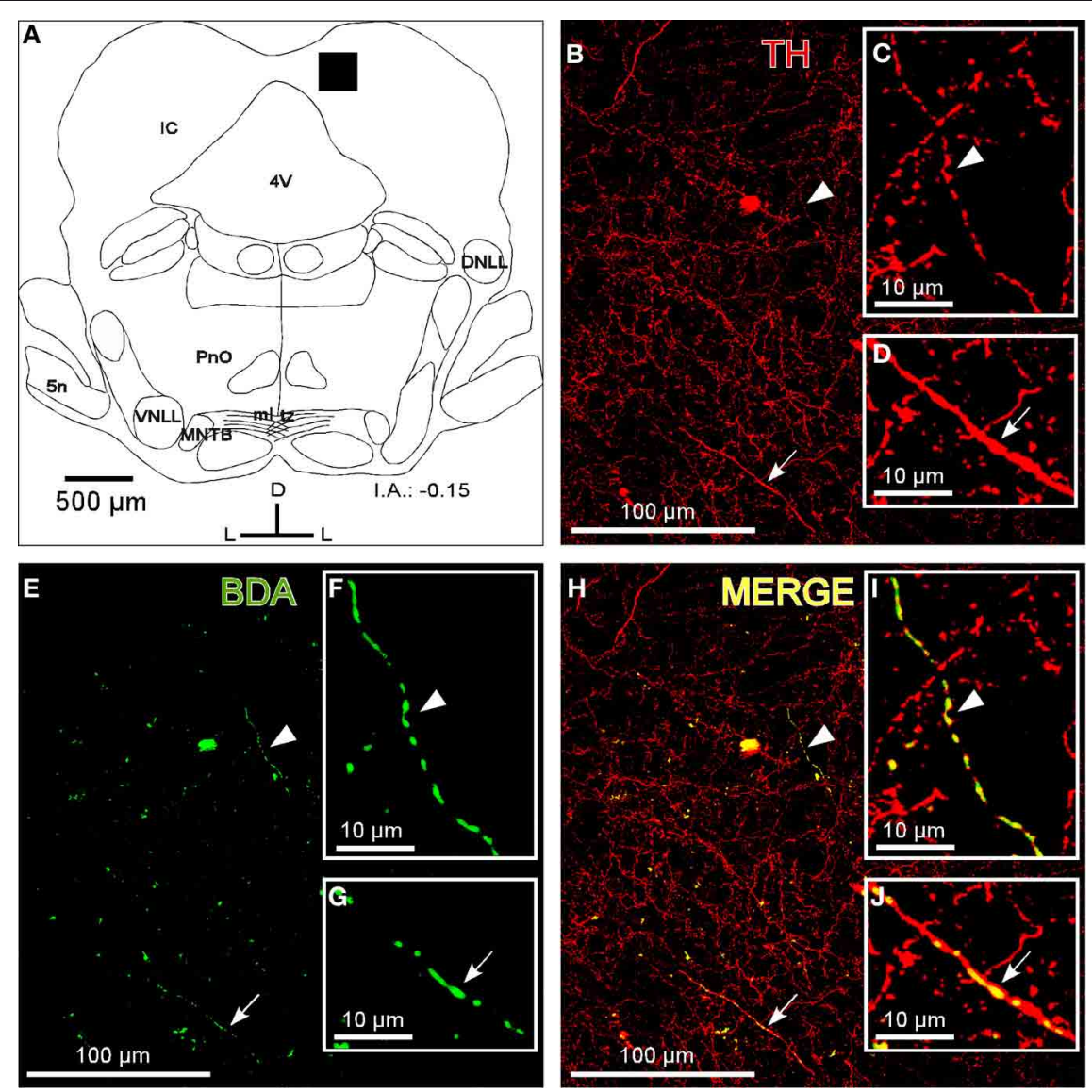

FIGURE 2 | The colocalization of locus coeruleus fibers with tyrosine hydroxylase (TH) in the dorsal cortex of the inferior colliculus (DCIC).

(A) A schematic representation of the coronal section in which the following confocal photomicrographs were taken. The black square depicts the area of the confocal images. (B) The confocal image shows TH-ir in DCIC (red). The arrow and arrowhead indicate representative $\mathrm{TH}$-labeled fibers magnified in $\mathbf{C}$ and $\mathbf{D}$, respectively. (E) The confocal image shows labeled fibers (green) after
BDA injection in the LC. The arrow and arrowhead indicate representative BDA-labeled fibers in the DCIC. The high magnification images of those fibers are shown in the insets $\mathbf{F}$ and $\mathbf{G}$. (H) The merged composition of the photomicrographs in $\mathbf{B}$ and $\mathbf{E}$ (yellow). The arrowhead and arrow indicate the colocalization of BDA and TH on the representative fibers that are magnified in $\mathbf{I}$ and $\mathbf{J}$, respectively. The interaural level for coronal placement is displayed in millimeters. See the provided list for abbreviations.
Similarly, we found dense DBH-ir in the entire IC in the control animals (Figure 7A). The caudal part presented the highest mean gray values, mainly in the DCIC and the CNIC. The fibers were uniformly distributed in the IC, and there was no significant difference in the distribution along the subdivisions $(p>$ $0.05)$. However, the reduction in DBH fibers in animals treated with DSP-4 was extremely severe (Figure 7B). The decrease of $\mathrm{DBH}$-ir took placed along the full length of the IC and in all its subdivisions, ranging from 89 to $97 \%$ (for all cases $p<0.01$ ) (Figures 7C,D, and E).

Based on these data, we conclude that DSP-4 removes the majority of the noradrenergic fibers in the IC, leaving only a few fibers spread in the different divisions of the IC and in the commissure.

\section{DISCUSSION}

Our findings suggest there is a direct projection from the LC to the IC. We found that this projection is mainly ipsilateral, reaching the DCIC, the LCIC and the commissure of the IC. In agreement with these results, previous studies have shown that the efferent projection from the LC to other nuclei, such as those from the somatosensory pathway, is mainly ipsilateral (Simpson et al., 1997). The neurochemical nature of these projections is noradrenergic, given that $\mathrm{TH}$ and $\mathrm{DBH}$ colocalize with the BDAlabeled fibers from the LC. We also found a dense immunoreactivity for TH in the IC, even when DSP-4 was administrated. Similar to our observations in the IC, the levels of $\mathrm{TH}$-immunoreactive axons within the hypothalamus, reticular formation, and brainstem motor nuclei appeared relatively unaffected following DSP-4 administration (Fritschy and Grzanna, 1989).

Consistent with Tong et al. (2005), our results also showed single-labeled fibers for $\mathrm{TH}$ and double-labeled fibers for $\mathrm{TH}$ and $\mathrm{DBH}$ in the IC. Moreover, phenylethanolamine-Nmethyltransferase, an enzyme use to identify adrenergic neurons and fibers, is not localized in the IC (Tong et al., 2005). In our study, TH-ir was not totally eliminated in the IC after the administration of DSP-4, which indicates the presence of a strong dopaminergic innervation within the IC. These data suggest that 

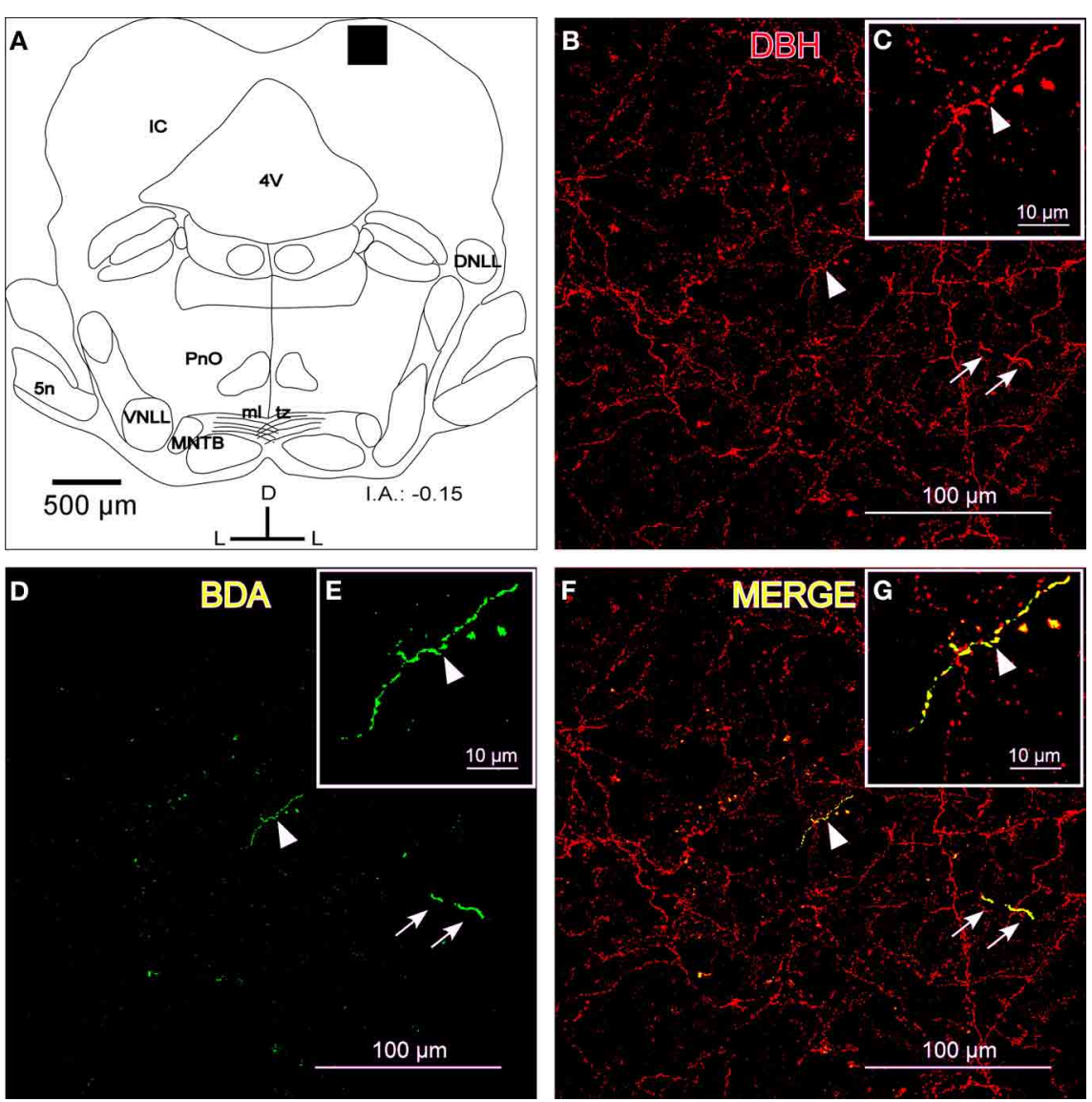

FIGURE 3 | The colocalization of locus coeruleus fibers with dopamine beta hydroxylase (DBH) in the dorsal cortex of the inferior colliculus (DCIC). (A) A schematic representation of the coronal section in which the following confocal photomicrographs were taken. The black square depicts the area of the confocal images. (B) A confocal image for DBH-ir (red). The arrows indicate a representative fiber in two parts, most likely due to the Z-axis frame, and the arrowhead shows another representative fiber that is magnified in C. (D) The confocal image shows labeled fibers (green) after BDA injection in the LC. The arrows indicate a representative fiber, and the arrowhead shows another representative fiber that is magnified in $\mathbf{E}$. (F) The merged composition between $\mathbf{B}$ and $\mathbf{D}$ (yellow). The arrowhead indicates a merged fiber (yellow) that is magnified in $\mathbf{G}$. The arrows show colocalization in another fiber (yellow). The interaural level for coronal placement is displayed in millimeters. See the provided list for abbreviations. both dopaminergic and noradrenergic systems are present in the IC. The source of the dopaminergic innervation might be from the substantia nigra (Garcia-Munoz et al., 1982; Takada et al., 1987; Kemel et al., 1988; Yasui et al., 1991; Deniau and Chevalier, 1992; Moriizumi et al., 1992; Yasui et al., 1995; Richards et al., 1997).

\section{THE LC-IC NORADRENERGIC INNERVATION AND THE EFFECTS OF DSP-4 ADMINISTRATION}

Noradrenergic fibers in the IC have been widely studied (Swanson and Hartman, 1975; Levitt and Moore, 1979; Olazàbal and Moore, 1989; Fritschy and Grzanna, 1990); however, the subnuclei distributions of the catecholaminergic innervations in the IC were uncharacterized. Kromer and Moore (1980) described a diffuse pattern of DBH-ir fibers in all the subdivisions of the IC, whereas Klepper and Herbert (1991) clearly indicated a subnuclei distribution of noradrenergic fibers in the IC based on immunohistochemistry for $\mathrm{DBH}$ and retrograde tract-tracing studies after injections into the IC.
Klepper and Herbert (1991) demonstrated that the noradrenergic projection to the IC largely arises from the LC and evidenced that these projections were bilateral with predominance $(2 / 1)$ toward the ipsilateral side. Because the injections sites in the IC were not considered in those retrograde tract-tracing studies (Klepper and Herbert, 1991), the projection field of the LC innervations in the IC remains unknown. Our anterograde track-tracing study verified that the LC-IC projection is mainly ipsilateral and further described the topographic distribution of the LC noradrenergic system in the rostrocaudal extent of the IC and its subdivisions.

Studies that describe the projections from the LC to the IC using anterograde tracers are often contradictory. The injection of lectin Phaseolus vulgaris leucoagglutinin (PHA-L) into the LC in combination with immunohistochemistry for $\mathrm{DBH}$ described axons that ran bilaterally into the inferior colliculi, but those LC axons appeared in a patch within sensory nuclei (Fritschy and Grzanna, 1990). Other authors, found very few labeled BDA fibers in the IC after BDA injections into the LC, with little difference 

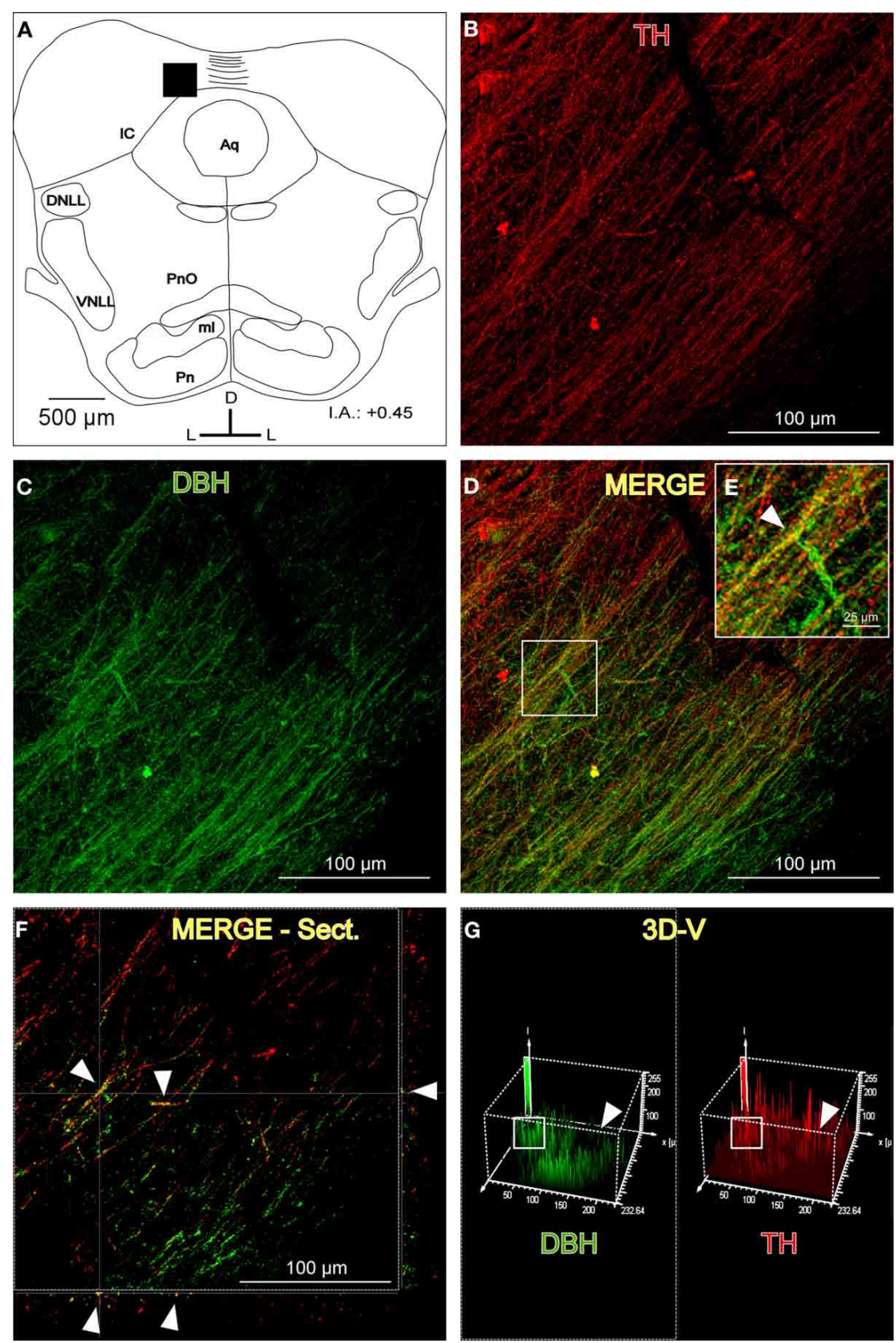

FIGURE 4 | The distribution of immunolabeled fibers for tyrosine hydroxylase (TH) and dopamine beta hydroxylase (DBH) in the inferior colliculus (IC). (A) Schematic representation of the coronal section in which the following confocal photomicrographs were taken. The black square depicts the exact placement of the confocal images, which were taken near the commissure. (B) The confocal image for TH-ir (red). (C) The confocal image for DBH-ir (green). (D) The merged composition between $\mathbf{B}$ and $\mathbf{C}$ (yellow). (E) A magnification of the white square in $\mathbf{D}$. The arrowhead indicates merged fibers (yellow). (F) An orthogonal view of a section of the confocal fibers. The arrowheads indicate merged fibers and their place in the Z-axis. (G) A three-dimensional view of the two channels. The arrowheads show the difference between the signals in the upper right part of the images where there are $\mathrm{TH}$-ir fibers (red) but not $\mathrm{DBH}$-ir, which leads to the conclusion that dopaminergic fibers (not noradrenergic) exist in the IC. The white square indicates the zone where the colocalization was found. There is a similar pattern of signal distribution. The interaural level for the coronal placement is displayed in millimeters. See the provided list for abbreviations. between the ipsi- and contralateral side (Mulders and Robertson, 2001).

On the other hand, there is another work that studied the afferent projections to the central nucleus of the IC in the rat that did not identify the LC as a source of afferents to this nucleus (Beyerl, 1978).

The LC is a nucleus with a considerably large caudo-rostral extension, and it has been described as a nucleus with a 

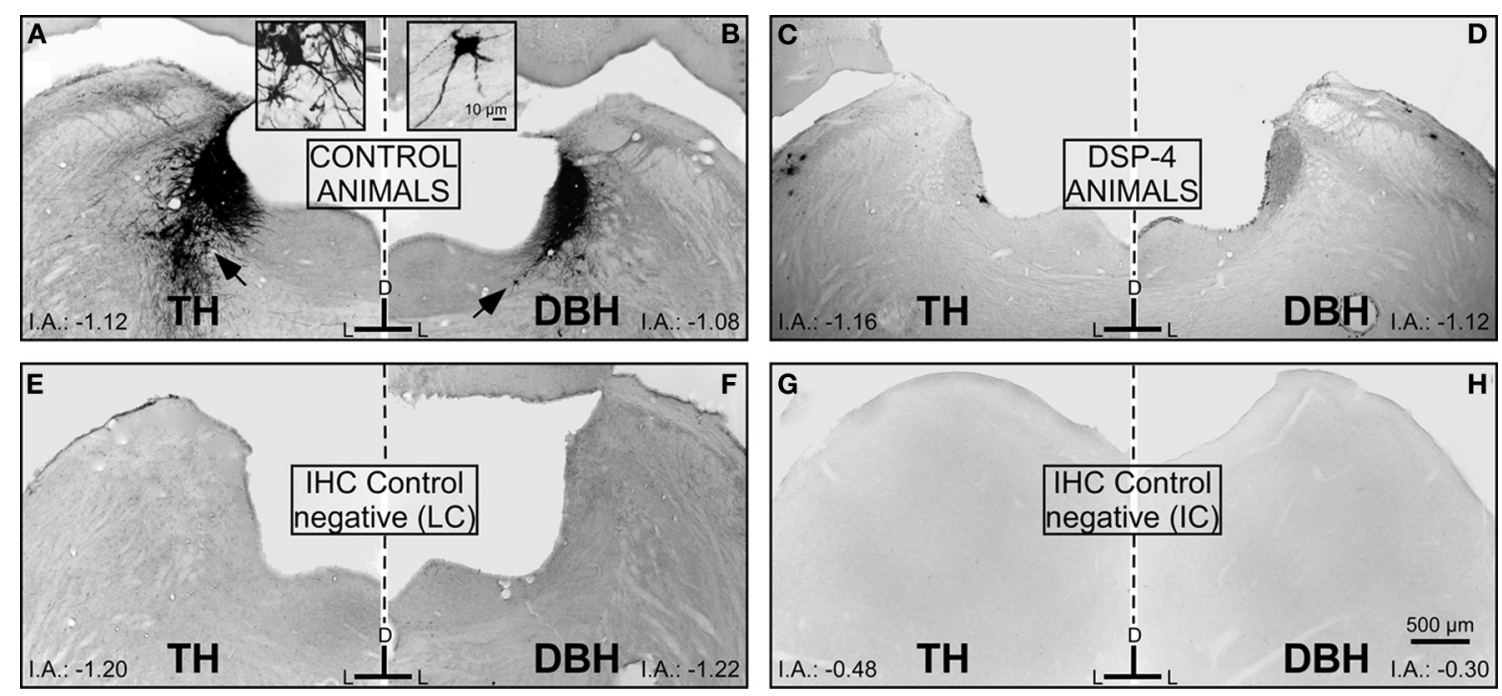

FIGURE 5 | The effects of DSP-4 administration in the locus coeruleus (LC) on immunohistochemistry (tyrosine hydroxylase and dopamine beta hydroxylase) compared to negative controls for the LC and the inferior colliculus (IC). (A) A photomicrograph of the locus coeruleus (LC) immunostained for tyrosine hydroxylase (TH) in a control animal. The black arrow represents a typical immunoreactive neuron, which is magnified in the inset. (B) A photomicrograph of the LC immunostained for dopamine-beta-hydroxylase $(\mathrm{DBH})$ in a control animal. The black arrow represents a typical immunoreactive neuron, which is magnified in the inset.
(C) A photomicrograph of the LC immunostained for TH in a DSP-4-treated animal. (D) A photomicrograph of the LC immunostained for DBH in a DSP-4-treated animal. Notice in this set of images how the immunoreactivity against the catecholaminergic enzymes is distinctly decreased compared to the controls. This reflects the immunoreactivity found in the IC in controls and treated animals. E,F, G, and $\mathbf{H}$ are images taken from the negative controls (no primary antibody added). The interaural level for the coronal placement is displayed in millimeters. See the provided list for abbreviations. heterogeneous neuronal population, differentiating its projections according to topographical criteria: axons directed to the forebrain and axons directed to the spinal cord (Mason and Fibiger, 1979; Grzanna and Molliver, 1980; Nagai et al., 1981; Loughlin et al., 1986). The contributions of each neuronal type to a broad efferent network are presented in detail in those studies, but there is no description of the projections to the IC.

In our study, we injected an anterograde tracer into small portions of the LC to avoid spreading to adjacent nuclei, which also project to the IC (for instance, the LDTg). Due to the small size of the BDA injection sites, only a fraction of all LC neurons was labeled in each case, resulting in light density axonal labeling. To determine the total field of the LC innervations in the IC, we destroyed the LC neurons and fibers using a highly selective neurotoxin, DSP-4, and then studied the distribution and density of the $\mathrm{TH}$ and $\mathrm{DBH}$ immunolabeled axons in the IC. DSP-4 administration models induce noradrenergic degeneration, exclusively affecting the noradrenergic fibers originating in the LC (Fritschy and Grzanna, 1989; Grzanna et al., 1989; Fritschy et al., 1991). Therefore, the densitometry analysis of TH and DBH-ir throughout the IC in DSP-4-treated and non-treated control rats provided us with valuable information regarding the extent and topographical distribution of the LC efferent network in the IC.

After DSP-4 treatment, we found a loss of TH immunoreactive fibers in the dorsal cortex and lateral cortex of the IC and nearly all $\mathrm{BDH}$ immunoreactive fibers. Additionally, there were a few remaining $\mathrm{DBH}$-ir that might belong to noradrenergic neurons distributed in the IC. These intrinsic noradrenergic neurons of the IC send descending projections to brainstem nuclei, including the LC (Tong et al., 2005; Freitas et al., 2006). In agreement with Fritschy and Grzanna (1989), our study verified the existence of two separate systems of noradrenergic fibers in the IC: the LC and non-coerulean noradrenergic systems. Our results further show that the sources of NA from non-coeruleus structures were much sparser than the lost of fibers from the LC. Therefore, our study verified that the LC system is the main source of NA in the IC and indicates that the fibers are distinctively distributed in the dorsal and lateral areas of the IC and the intercollicular commissure. These results might contribute to the understanding of the functional role of the IC-LC projection.

\section{FUNCTIONAL IMPLICATIONS}

Novelty detection is concerned with recognizing inputs that differ in some way from previous inputs. In all sensorial modalities, an unexpected and novel perception is highly effective in attracting and focusing attention. Both the IC and the LC play a major role in novelty detection. The IC constitutes an obligatory and highly integrative relay for the ascending and descending auditory pathways and also process multimodal information, including visual cues (Cooper and Young, 1976; Elverland, 1978; Morest and Oliver, 1984; Faye-Lund and Osen, 1985; Saldana et al., 1996). IC neurons respond selectively to novel sounds and show stimulusspecific adaptation (Pérez-González et al., 2005; Malmierca et al., 2009). Similarly, the LC neurons integrate multimodal information and increase their firing rates following the presentation of novel stimuli (Foote et al., 1980; Grant et al., 1988). Therefore, both midbrain nuclei (IC and LC) are essential for eliciting an 


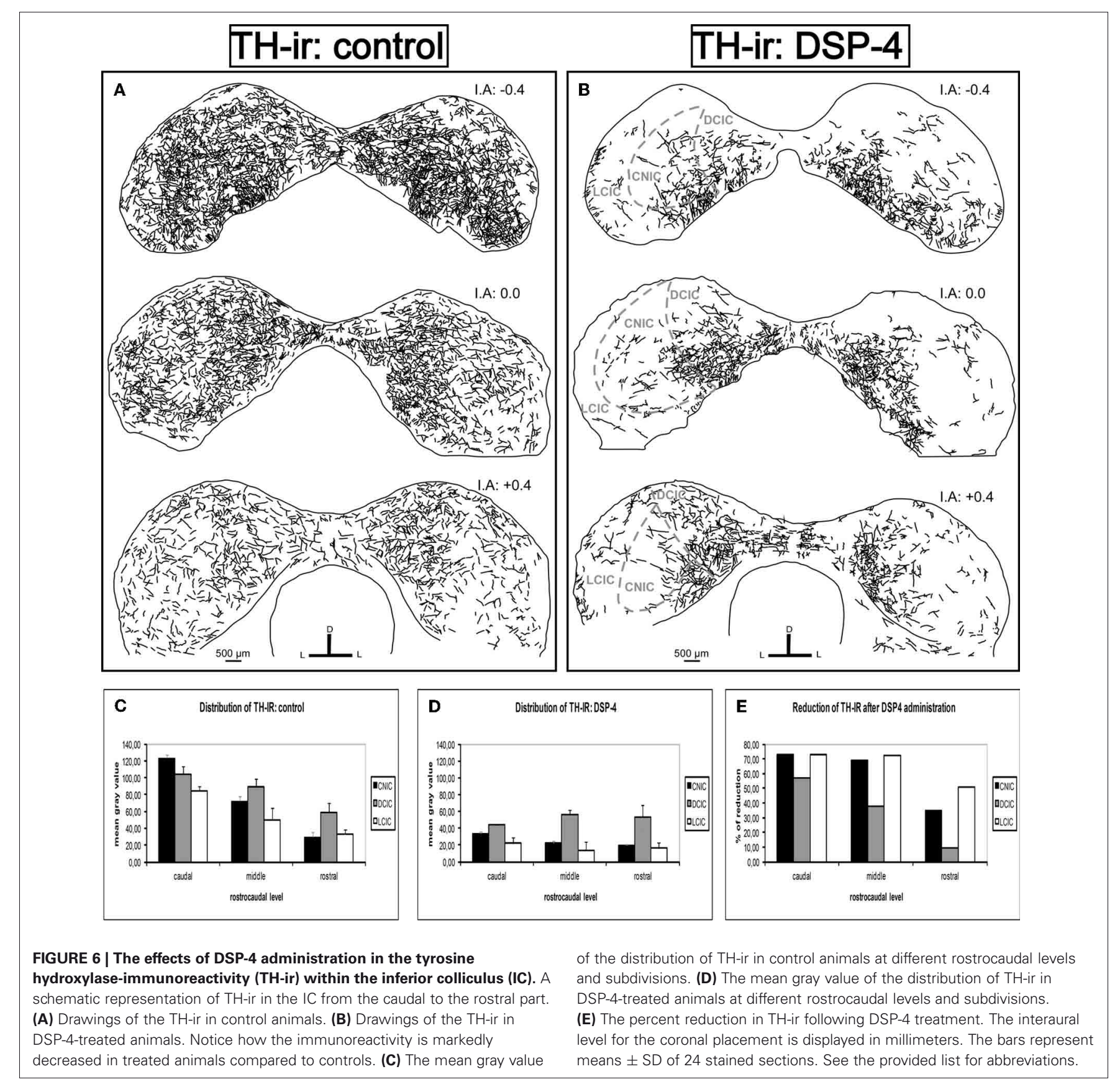

orienting response. Our study demonstrates a noradrenergic projection from the LC to the IC, suggesting a functional correlation between the two nuclei. In fact, the main target areas and projection pattern of the LC fibers within the IC are precisely the same IC areas where the population of novelty neurons is located.

In comparison with the neurons in the central nucleus of the IC, the neurons in the dorsal and lateral IC show a strong stimulus-specific adaptation that might contribute to auditory scene analysis, novelty detection, and selective attention (PérezGonzález et al., 2005; Malmierca et al., 2009; Lumani and Zhang, 2010). Interestingly, our results showed that the noradrenergic projection from the LC projects to the dorsal and lateral cortex of the IC and, to a lesser extent, to the central nucleus of the IC. Modulators like NA might participate in stimulus-specific adaption given that NA blocks both $\mathrm{Ca}^{2+}$ - and $\mathrm{Na}^{+}$-dependent $\mathrm{K}^{+}$ currents (Foehring et al., 1989). Such $\mathrm{K}^{+}$currents underlie at least part of the cortical auditory adaptation in awake rats (Abolafia et al., 2011). This suggests that the noradrenergic LC-IC projection might contribute to the information that this population of IC neurons integrates for novelty detection.

As we have shown in the IC, the cochlear nucleus and the superior olivary complex also receive noradrenergic inputs from the LC (Klepper and Herbert, 1991; Ebert, 1996; Mulders and Robertson, 2001; Thompson, 2003). Therefore, all the major relay 


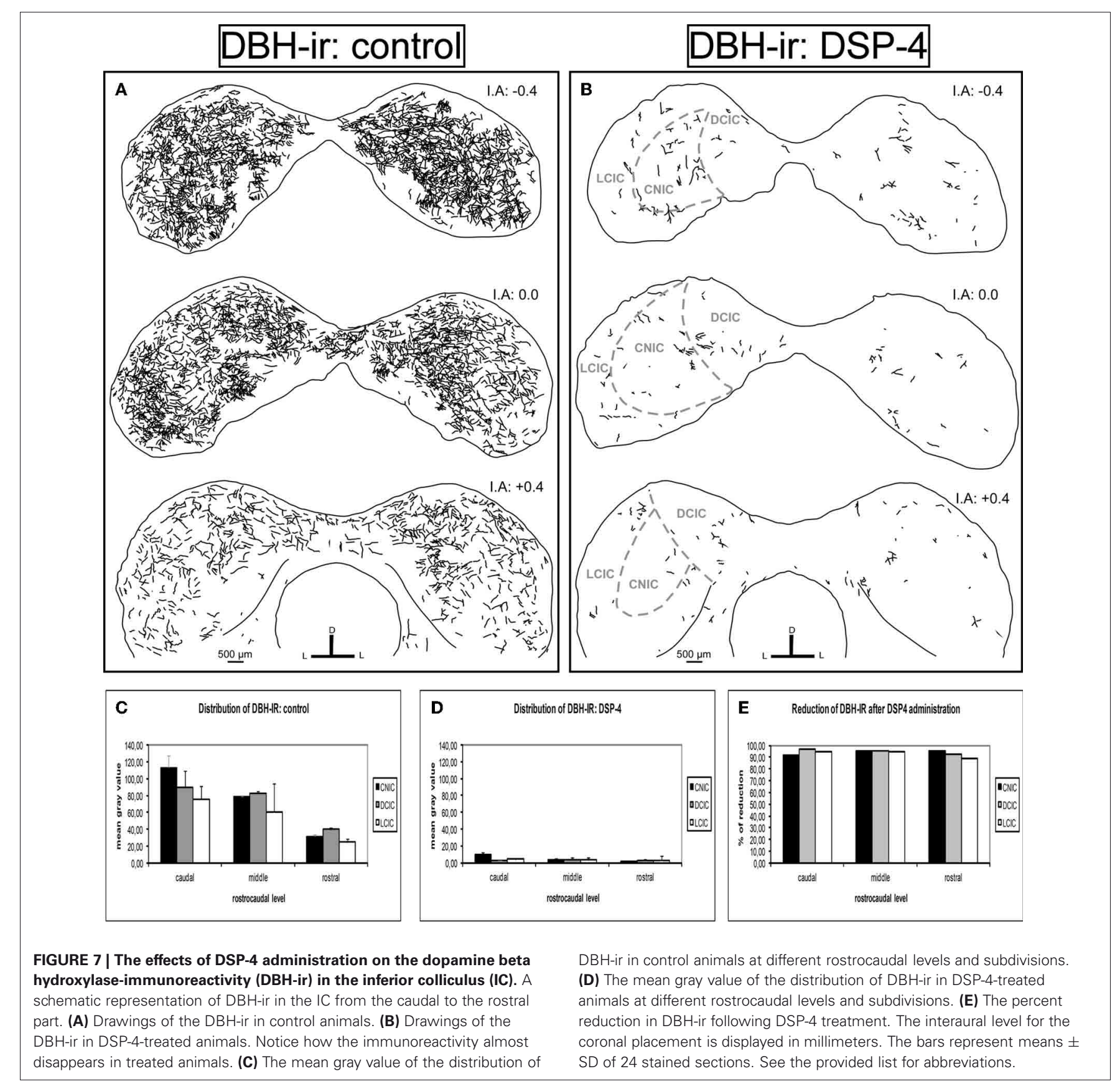

nuclei for auditory information are influenced by the neuromodulator NA. In the context of the NA regulation of behaviors, the activation of the LC noradrenergic system is known to increase attentional processes and influence the target circuits involved in arousal states, learning and memory, alertness, and vigilance (Aston-Jones et al., 1991; Woodward et al., 1991; Berridge and Waterhouse, 2003; Lapiz and Morilak, 2006). This leads us to question how the LC noradrenergic system affects auditory perception and how auditory processing is carried out under different behavioral circumstances. In auditory cue detection, a recent study showed that $\alpha-2$ adrenergic stimulation is effective in modulating accuracy and response latencies in non-stressed animals
(Brown et al., 2012). This suggests that NA not only induces the arousal state to initiate attentional processing but also suggests a more specific role for NA in auditory signal versus non-signal discrimination (Brown et al., 2012). Because the cortex of the IC is particularly rich in $\alpha-2$ adrenergic receptors (Probst et al., 1984), it is essential to know the target areas of the LC noradrenergic system within the IC. Our study indicates that the LC noradrenergic system massively innervates the dorsal and lateral portions of the IC and revealed that these innervations are significantly impaired in DSP-4-treated rats. These results might contribute to the design of new experiments focused on the role of $\alpha$ adrenergic receptors in the dorsal and lateral IC. 
Additionally, the LC projects to the cochlear root nucleus, another midbrain nucleus, which processes unexpected acoustic stimuli and mediates auditory alerting and escape behaviors, such as the acoustic startle reflex (Lee et al., 1996; López et al., 1999; Gómez-Nieto et al., 2008b). The cochlear root nucleus projects to the facial motor neurons that promote pinna movements toward unexpected, loud sounds (Horta-Júnior et al., 2008) and also receives inputs from the IC via a short descending auditory pathway that relays in the ventral nucleus of the trapezoid body (Gómez-Nieto et al., 2008a). Because the IC plays a role in binaural inhibition and is critical for the modulation of the acoustic startle reflex by auditory stimuli (reviewed in Fendt et al., 2001), it is of great interest to study the connections between the IC, LC, and the cochlear root nucleus. The main goal of the present study was to re-examine the projections from the LC to IC in intact and DSP-4 treated rats. Our

\section{REFERENCES}

Abolafia, J. M., Vergara, R., Arnold, M. M., Reig, R., and Sanchez-Vives, M. V. (2011). Cortical auditory adaptation in the awake rat and the role of potassium currents. Cereb. Cortex 21, 977-990.

Aston-Jones, G. (2004). "Locus coeruleus, A5 and A7 noradrenergic cell groups," in The Rat Nervous System, ed G. Paxinos (San Diego, CA: Elsevier), 259-294.

Aston-Jones, G., Chiang, C., and Alexinsky, T. (1991). Discharge of noradrenergic locus coeruleus neurons in behaving rats and monkeys suggests a role in vigilance. Prog. Brain Res. 88, 501-520.

Axelrod, J., Kopin, I. J., and Waser, K. A. (1969). The uptake, storage, release and metabolism of noradrenaline in sympathetic nerves. Prog. Brain Res. 31, 21-32.

Berridge, C. W., and Waterhouse, B. D. (2003). The locus coeruleusenoradrenergic system: modulation of behavioral state and statedependent cognitive processes. Brain Res. Rev. 42, 33-84.

Beyerl, B. D. (1978). Afferent projections to the central nucleus of the inferior colliculus in the rat. Brain Res. 145, 209-223.

Brown, D. C. 2nd, Co, M. S., Wolff, R. C., and Atzori, M. (2012). $\alpha$-Adrenergic receptors in auditory cue detection: $\alpha(2)$ receptor blockade suppresses false alarm responding in the rat. Neuropharmacology 62, 2177-2182.

Ciaranello, R. D., Wooten, F. G., and Axelrod, J. (1976). Regulation of rat adrenal dopamine B-hydroxylase. II. Receptor interaction in the regulation of enzyme synthesis and degradation. Brain Res. 113, 349-362.

Conti, L. H., and Foote, S. L. (1996). Reciprocal cross-desensitization of locus coeruleus electrophysiological responsivity to corticotropinreleasing factor and stress. Brain Res. 722, 19-29.

Cornwell-Jones, C. A., Palfai, T., Krasenbaum, D., Byer, E. Jr., Clark, R., and Kinnard, K. (1992). Housing influences exploration and social interaction of control and DSP4-treated rats. Physiol. Behav. 52, 271-276.

Couto, L. B., Moroni, C. R., Ferreira, C. M., Elias-Filho, D. H., Parada, C., Pel, I. R., and Coimbra, N. C. (2006). Descriptive and functional neuroanatomy of locus coeruleusnoradrenaline-containing neurons involvement in bradykinin-induced antinociception on principal sensory trigeminal nucleus. J. Chem. Neuroanat. 32, 28-45.

Cooper, M. H., and Young, P. A. (1976). Cortical projections to the inferior colliculus of the cat. Exp. Neurol. 51, 488-502.

Curtis, A. L., Drolet, G., and Valentino, R. J. (1993). Hemodynamic stress activates locus coeruleus neurons of unanesthetized rats. Brain Res. Bull. 31, 737-744.

Dawe, G. S., Huff, K. D., Vandergriff, J. L., Sharp, T., O’Neill, M. J., and Rasmussen, K. (2001). Olanzapine activates the rat locus coeruleus: in vivo electrophysiology and c-Fos immunoreactivity. Biol. Psychiatry 50, 510-520.

del C. Gonzalez, M. M., Debilly, G., Valatx, J. L., and Jouvet, M. (1995). Sleep increase after immobilization stress: role of the noradrenergic locus coeruleus system in the rat. Neurosci. Lett. 202, 5-8.

Delini-Stula, A., Mogilnicka, E., Hunn, C., and Dooley, D. J. (1984). Novelty-oriented behavior in the rat after selective damage of locus coeruleus projections by DSP-4,

results demonstrate that the noradrenergic projection from the LC to the IC was drastically impaired after DSP-4 administration. This implies that DSP-4 might be used as a powerful tool to study the functional role of the projections from the LC to midbrain nuclei, such as the IC and the cochlear root nucleus, that are involved in novelty detection, orienting response, and alerting behaviors.

\section{ACKNOWLEDGMENTS}

We would like to express gratitude to the Spanish Ministry of Science and Innovation, (MICINN, \# BFU2010-17754) and to the Sao Paulo State Research Foundation (FAPESP proc. 2008/02771-6) for their financial support. Moreover, we thank the AUIP (Programa de Becas de Movilidad Académica 2010-2011) for facilitating a fellowship in Brazil in which some parts of the experiments above took place.

a new noradrenergic neurotoxin. Pharmacol. Biochem. Behav. 20, 613-618.

Deniau, J. M., and Chevalier, G. (1992). The lamellar organization of the rat substantia nigra pars reticulata: distribution of projection neurons. Neuroscience 46, 361-377.

Ebert, U. (1996). Noradrenaline enhances the activity of cochlear nucleus neurons in the rat. Eur. J. Neurosci. 8, 13006-13014.

Elverland, H. H. (1978). Ascending and intrinsic projections of the superior olivary complex in the cat. Exp. Brain Res. 32, 117-134.

Faye-Lund, H., and Osen, K. K. (1985). Anatomy of the inferior colliculus in rat. Anat. Embryol. 171, 1-20.

Fendt, M., Li, L., and Yeomans, J. S (2001). Brain stem circuits mediating prepulse inhibition of the startle reflex. Psychopharmacology (Berl.) 156, 216-224.

Foehring, R. C., Schwindt, P. C., and Crill, W. E. (1989). Norepinephrine selectively reduces slow $\mathrm{Ca} 2+-$ and $\mathrm{Na}+$-mediated $\mathrm{K}+$ currents in cat neocortical neurons. $J$. Neurophysiol. 61, 245-256.

Foote, S. L., Aston-Jones, G., and Bloom, F. E. (1980). Impulse activity of locus coeruleus neurons in awake rats and monkeys is a function of sensory stimulation and arousal. Proc. Natl. Acad. Sci. U.S.A. 77, 3033-3037.

Freitas, R. L., Ferreira, C. M. R., Ribeiro, S. J., Carvalho, A. D., Elias-Filho, D. H., Garcia-Cairasco, N., and Coimbra, N. C. (2006) Intrinsic neural circuits between dorsal midbrain neurons that control fear-induced responses and seizure activity and nuclei of the pain inhibitory system elaborating postictal antinociceptive processes: a functional neuroanatomical and neuropharmacological study. Exp. Neurol. 191, 225-242.

Fritschy, J. M., and Grzanna, R. (1989). Immunohistochemical analysis of the neurotoxic effects of DSP4 identifies two populations of noradrenergic axon terminals. Neuroscience 30, 181-197.

Fritschy, J. M., and Grzanna, R. (1990). Distribution of locus coeruleus axons within the rat brainstem demonstrated by Phaseolus vulgaris leucoagglutinin anterograde tracing in combination with dopaminebeta-hydroxylase immunofluorescence. J. Comp. Neurol. 293, 616-631.

Fritschy, J. M., Grzanna, R., and Pompeiano, C. D. (1991). Chapter 20 Selective effects of DSP-4 on locus coeruleus axons: are there pharmacologically different types of noradrenergic axons in the central nervous system? Prog. Brain Res. 88, 257-268.

Garcia-Munoz, M., Patino, P., Aguilar, R., and Arbuthnott, G. (1982). Participation of projections from substantia nigra reticulata to the lower brain stem in turning behavior. Exp. Neurol. 78, 380-390.

Gómez-Nieto, R., Rubio, M. E., and López, D. E. (2008a). Cholinergic input from the ventral nucleus of the trapezoid body to cochlear root neurons in rats. J. Comp. Neurol. 506, 452-468.

Gómez-Nieto, R., Horta-Junior, J. A. C., Castellano, O., Herrero-Turrión, M. J., Rubio, M. E., and López, D. E. (2008b). Neurochemistry of the afferents to the rat cochlear root nucleus: possible synaptic modulation of the acoustic startle. Neuroscience 154, 51-64.

Grant, S. J., Aston-Jones, G., and Redmond, D. E. (1988). Responses of primate locus 'coeruleus neurons' 
to 'simple and complex' sensory 'stimuli. Brain Res. Bull. 21, 401-410.

Grzanna, R., Berger, U., Fritschy, J. M., and Geffard, M. (1989). Acute action of DSP-4 on central norepinephrine axons: biochemical and immunohistochemical evidence for differential effects. J. Histochem. Cytochem. 37, 1435-1442.

Grzanna, R., and Molliver, M. E. (1980). The locus coeruleus in the rat: an immunohistochemical delineation. Neuroscience 5, 21-40.

Guyenet, P. G. (1980). The coeruleospinal noradrenergic neurons: anatomical and electrophysiological studies in the rat. Brain Res. 189, 121-133.

Hancock, M. B. (1982). DAB-Nikel substrate for the differential immunoperoxidase staining of nerve fibers and fiber terminals. J. Histochem. Cytochem. 30, 578.

Harro, J., Oreland, L., Vasar, E., and Bradwejn, J. (1995). Impaired exploratory behaviour after DSP-4 treatment in rats: implications for the increased anxiety after noradrenergic denervation. Eur. Neuropsychopharmacol. 5, 447-455.

Heldt, S. A., and Falls, W. A. (2003). Destruction of the inferior colliculus disrupts the production and inhibition of fear conditioned to an acoustic stimulus. Behav. Brain Res. 144, 175-185.

Horta-Júnior, J. A., López, D. E., Alvarez-Morujo, A. J., and Bittencourt, J. C. (2008). Direct and indirect connections between cochlear root neurons and facial motor neurons: pathways underlying the acoustic pinna reflex in the albino rat. J. Comp. Neurol. 507, 1763-1779.

Hsu, S. M., and Soban, E. (1982). Color modification of diaminobenzidine (DAB) precipitation by metallic ions and its application for double immunohistochemistry. J. Histochem. Cytochem. 30, 1079-1082.

Imbe, H., Okamoto, K., Donishi, T., Kawai, S., Enoki, K., Senba, E., and Kimura, A. (2009). Activation of ERK in the locus coeruleus following acute noxious stimulation. Brain Res. 1263, 50-57.

Jordan, W. P., and Leaton, R. N. (1982). Startle habituation in rats after lesions in the brachium of the inferior colliculus. Physiol. Behav. $28,253-258$

Kemel, M. L., Desban, M., Gauchy, C., Glowinski, J., and Besson, M. J. (1988). Topographical organization of efferent projections from the cat substantia nigra pars reticulata. Brain Res. 455, 307-323.

Kim, M. A., Lee, H. S., Lee, B. Y., and Waterhouse, B. D. (2004). Reciprocal connections between subdivisions of the dorsal raphe and the nuclear core of the locus coeruleus in the rat. Brain Res. 1026, 56-67.

Klepper, A., and Herbert, H. (1991). Distribution and origin of noradrenergic and serotonergic fibers in the cochlear nucleus and inferior colliculus of the rat. Brain Res. 557, 190-201.

Kromer, L. F., and Moore, R. Y. (1980). A study of the organization of the locus coeruleus projections to the lateral geniculate nuclei in the albino rat. Neuroscience 5, 255-271.

Lapiz, M. D. S., and Morilak, D. A. (2006). Noradrenergic modulation of cognitive function in rat medial prefrontal cortex as measured by attentional set shifting capability. Neuroscience 137, 1039-1049.

Lechner, S. M., Curtis, A. L., Brons, R., and Valentino, R. J. (1997). Locus coeruleus activation by colon distention: role of corticotropinreleasing factor and excitatory amino acids. Brain Res. 756, 114-124.

Lee, Y., López, D. E., Meloni, E. G., and Davis, M. (1996). A primary acoustic startle pathway: obligatory role of cochlear root neurons and the nucleus reticularis pontis caudalis. J. Neurosci. 16, 3775-3789.

Leitner, D. S., and Cohen, M. E. (1985). Role of the inferior colliculus in the inhibition of acoustic startle in the rat. Physiol. Behav. 34, 65-70.

Levitt, P., and Moore, R. Y. (1979). Origin and organization of brainstem catecholamine innervations in rat. J. Comp. Neurol. 186, 505-528.

Li, L., Korngut, L. M., Frost, B. J., and Beninger, R. J. (1998). Prepulse inhibition following lesions of the inferior colliculus: prepulse intensity functions. Physiol. Behav. 65, 133-139.

Li, L., and Yeomans, J. S. (2000). Using intracranial electrical stimulation to study the timing of prepulse inhibition of the startle reflex. Brain Res. Brain Res. Protoc. 5, 67-74.

Li, L., and Yue, Q. (2002). Auditory gating processes and binaural inhibition in the inferior colliculus. Hear. Res. 168, 98-109.

Liu, L., Tsuruoka, M., Maeda, M., Hayashi, B., Wang, X., and Inoue, T. (2008). Descending modulation of visceral nociceptive transmission from the locus coeruleus/subcoeruleus in the rat. Brain Res. Bull. 76, 616-625.

López, D. E., Saldana, E., Nodal, F. R., Merchán, M. A., and Warr, W. B.
(1999). Projections of cochlear root neurons, sentinels of the auditory pathway in the rat. J. Comp. Neurol. $415,160-174$.

Loughlin, S. E., Foote, S. L., and Grzanna, R. (1986). Efferent projections of nucleus locus coeruleus: morphologic subpopulations have different efferent targets. Neuroscience 18, 307-319.

Lumani, A., and Zhang, H. (2010). Responses of neurons in the rat's dorsal cortex of the inferior colliculus to monaural tone bursts. Brain Res. 1351, 115-129.

Ma, S., Mifflin, S. W., Cunningham, J. T., and Morilak, D. A. (2008) Chronic intermittent hypoxia sensitizes acute hypothalamic-pituitaryadrenal stress reactivity and Fos induction in the rat locus coeruleus in response to subsequent immobilization stress. Neuroscience 154 1639-1647.

Malmierca, M. S., Cristaudo, S., Pérez-González, D., and Covey, E. (2009). Stimulus-specific adaptation in the inferior colliculus of the anesthetized rat. J. Neurosci. 29, 5483-5493.

Mason, S. T., and Fibiger, H. C. (1979). Regional topography within noradrenergic locus coeruleus as revealed by retrograde transport of horseradish peroxidase. J. Comp. Neurol. 187, 703-724.

Miao-Kun, S. (1995). Central neura organization and control of sympathetic nervous system in mammals. Prog. Neurobiol. 47, 157-233.

Moriizumi, T., Leduc-Cross, B., Wu, J. Y., and Hattori, T. (1992). Separate neuronal populations of the rat substantia nigra pars lateralis with distinct projection sites and transmitter phenotypes. Neuroscience 46 711-720.

Morest, D. K., and Oliver, D. L. (1984). The neuronal architecture of the inferior colliculus in the cat: defining the functional anatomy of the auditory midbrain. J. Comp. Neurol. 222, 209-236.

Mulders, W. H. A. M., and Robertson, D. (2001). Origin of the noradrenergic innervation of the superior olivary complex in the rat. J. Chem. Neuroanat. 21, 313-322.

Nagai, T., Satoh, K., Imamoto, K., and Maeda, T. (1981). Divergent projections of catecholamine neurons of the locus coeruleus as revealed by fluorescent retrograde double labeling technique. Neurosci. Lett. 23, 117-123.

Nobre, M. J., Sandner, G., and Brandao, M. L. (2003). Enhancement of acoustic evoked potentials and impairment of startle reflex induced by reduction of GABAergic control of the neural substrates of aversion in the inferior colliculus. Hear. Res. 184, 82-90.

Olazàbal, U. E., and Moore, J. K. (1989). Nigrotectal projection to the inferior colliculus: horseradish perosidase transport and tyroxine hydroxylase immunohystochemical studies in rats, cats and bats. J. Comp. Neurol. 282, 98-118.

Page, M. E., Akaoka, H., AstonJones, G., and Valentino, R. J. (1992). Bladder distention activates noradrenergic locus coeruleus neurons by an excitatory amino acid mechanism. Neuroscience 51, 555-563.

Paxinos, G., and Watson, C. (2005). The Rat Brain in Stereotaxic Coordinates: The New Coronal set-161 Diagrams, 5th edn. San Diego, CA: Academic Press.

Pérez-González, D., Malmierca, M. S., and Covey, E. (2005). Novelty detector neurons in the mammalian auditory midbrain. Eur. J. Neurosci. 22, 2879-2885.

Potter, L. T., Axelrod, J., and Kopin, I. J. (1962). Differential binding and release of norepinephrine and tachyphylaxis. Biochem. Pharmacol. 11, 254-256.

Probst, A., Cortés, R., and Palacios, J. M. (1984). Distribution of $\alpha 2$ adrenergic receptors in the human brainstem: an autoradiographic study using $[3 \mathrm{H}] \mathrm{p}$-aminoclonidine. Eur. J. Pharmacol. 106, 477-488.

Ranson, R. N., Gaunt, K., Santer, R. M., and Watson, A. H. D. (2003). The effects of ageing and of DSP4 administration on the micturition characteristics of male Wistar rats. Brain Res. 988, 130-138.

Reimer, A. E., Oliveira, A. R., and Brandao, M. L. (2008). Selective involvement of GABAergic mechanisms of the dorsal periaqueductal gray and inferior colliculus on the memory of the contextual fear as assessed by the fear potentiated startle test. Brain Res. Bull. 76, 545-550.

Richards, C. D., Shiroyama, T., and Kitai, S. T. (1997). Electrophysiological and immunocytochemical characterization of GABA and dopamine neurons in the substantia nigra of the rat. Neuroscience 80, 545-557.

Ross, S. B. (1985). DSP-4 and behavioural experiments. Pharmacol. Sci. 6, 237.

Ross, S. B., Johansson, J. G., Lindborg, B., and Dahlbom, R. (1973). Cyclizing compounds. I. Tertiary N-(2-bromobenzyl)$\mathrm{N}$-haloalkylamines with adrenergic 
blocking action. Acta Pharm. Suec. $10,29-42$.

Saldana, E., Feliciano, M., and Mugnaini, E. (1996). Distribution of descending projections from primary auditory neocortex to inferior colliculus mimics the topography of intracollicular projections. J. Comp. Neurol. 371, 15-40.

Satake, S., Yamada, K., Melo, L. L., and Barbosa, S. R. (2012). Effects of microinjections of apomorphine and haloperidol into the inferior colliculus on prepulse inhibition of the acoustic startle reflex in rat. Neurosci. Lett. 509, 60-63.

Silva, R. C. B., Sandner, G., and Brandao, M. L. (2005). Unilateral electrical stimulation of the inferior colliculus of rats modifies the prepulse modulation of the startle response (PPI): effects of ketamine and diazepam. Behav. Brain Res. 160, 323-330.

Simpson, K. L., Altman, D. W., Wang, L., Kirifides, M. L., Lin, R. C., and Waterhouse, B. D. (1997). Lateralization and functional organization of the locus coeruleus projection to the trigeminal somatosensory pathway in rat. J. Comp. Neurol. 385, 135-147.

Singewald, N., Zhou, G. Y., and Schneider, C. (1995). Release of excitatory and inhibitory amino acids from the locus coeruleus of conscious rats by cardiovascular stimuli and various forms of acute stress. Brain Res. 704, 42-50.

Snyder, S. H., Glowinski, J., and Axelrod, J. (1965). The storage of norepinephrine and some of its derivatives in brain synaptosomes. Life Sci. 4, 797-807.

Spyraki, C., Arbuthnott, G. W., and Fibiger, H. C. (1982). The effect of DSP-4 on some positively reinforced operant behaviors in the rat. Pharmacol. Biochem. Behav. 16, 197-202.

Swanson, L. W., and Hartman, B. K. (1975). The central adrenergic system: an immunofluorescence study of the location of cell bodies and their efferent connections in the rat utilizing dopamine- $\beta$ hydroxylase as a marker. J. Comp. Neurol. 163, 467-506.

Takada, M., Li, Z. K., and Hattori, T. (1987). A note on the projections of pars compacta neurons within pars reticulata of the substantia nigra in the rat. Brain Res. Bull. 18, 285-290.

Thompson, A. M. (2003). Pontine sources of norepinephrine in the cat cochlear nucleus. J. Comp. Neurol. 457, 374-383.

Tong, L., Altschuler, R. A., and Holt, A. G. (2005). Tyrosine hydroxylase in rat auditory midbrain: distribution and changes following deafness. Hear. Res. 206, 28-41.

Toshihiro, M. (2000). The locus coeruleus: history. J. Chem. Neuroanat. $18,57-64$.

Tsuruoka, M., Tamaki, J., Maeda, M., Hayashi, B., and Inoue, T. (2011). The nucleus locus coeruleus/subcoeruleus contributes to antinociception during freezing behavior following the air-puff startle in rats. Brain Res. 1393, 52-61.

Valentino, R. J., Foote, S. L., and Aston-Jones, G. (1983). Corticotropin-releasing factor activates noradrenergic neurons of the locus coeruleus. Brain Res. 270 363-367.

Valentino, R. J., and Van Bockstaele, E. (2008). Convergent regulation of locus coeruleus activity as an adaptive response to stress. Eur. J. Pharmacol. 583, 194-203.

Valentino, R. J., Van Bockstaele, E. J., and Steckler, T. (2005). "Chapter 4.4 Functional interactions between stress neuromediators and the locus coeruleus-norepinephrine system,' in Techniques in the Behavioral and Neural Sciences, Part 1, Vol. 15, eds J. M. H. M. Reul, N. H. Kalin, and T. Steckler (Philadelphia, PA: Elsevier), 465-486.

van den Buuse, M., Lambert, G., Fluttert, M., and Eikelis, N. (2001). Cardiovascular and behavioural responses to psychological stress in spontaneously hypertensive rats effect of treatment with DSP-4 Behav. Brain Res. 119, 131-142.

Viisanen, H., and Pertovaara, A. (2007). Influence of peripheral nerve injury on response properties of locus coeruleus neurons and coeruleospinal antinociception in the rat. Neuroscience 146, 1785-1794.

Woodward, D. J., Moises, H. C., Waterhouse, B. D., Yeh, H. H., and Cheun, J. E. (1991). Modulatory actions of norepinephrine on neura circuits. Adv. Exp. Med. Biol. 287, 193-208.

Yasui, Y., Nakano, K., Kayahara, T., and Mizuno, N. (1991). Nondopaminergic projections from the substantia nigra pars lateralis to the inferior colliculus in the rat. Brain Res. 559, 139-144.
Yasui, Y., Tsumori, T., Ando, A., and Domoto, T. (1995). Demonstration of axon collateral projections from the substantia nigra pars reticulata to the superior colliculus and the parvicellular reticular formation in the rat. Brain Res. 674 122-126.

Zaczek, R., Fritschy, J. M., Culp, S., De Souza, E. B., and Grzanna, R. (1990). Differential effects of DSP-4 on noradrenaline axons in cerebral cortex and hypothalamus may reflect heterogeneity of noradrenaline uptake sites. Brain Res. 522, 308-314.

Conflict of Interest Statement: The authors declare that the research was conducted in the absence of any commercial or financial relationships that could be construed as a potential conflict of interest.

Received: 30 March 2012; accepted: 12 June 2012; published online: 28 June 2012.

Citation: Hormigo S, Horta Júnior JAC, Gómez-Nieto R and López DE (2012) The selective neurotoxin DSP-4 impairs the noradrenergic projections from the locus coeruleus to the inferior colliculus in rats. Front. Neural Circuits 6:41. doi: 10.3389/fncir.2012.00041

Copyright (C) 2012 Hormigo, Horto Júnior, Gómez-Nieto and López. This is an open-access article distributed under the terms of the Creative Commons Attribution Non Commercial License, which permits non-commercial use, distribution, and reproduction in other forums, provided the original authors and source are credited. 